\title{
LOCAL TRANSFORMATIONS BETWEEN SOME NONLINEAR DIFFUSION EQUATIONS
}

\author{
J. R. KING ${ }^{1}$
}

(Received 1 November 1990; revised 14 January 1991)

\begin{abstract}
We derive local transformations mapping radially symmetric nonlinear diffusion equations with power law or exponential diffusivities into themselves or into other equations of a similar form. Both discrete and continuous transformations are considered. For the cases in which a continuous transformation exists, many additional forms of group-invariant solution may be constructed; some of these solutions may be written in closed form. Related invariance properties of some multidimensional diffusion equations are also exploited.
\end{abstract}

\section{Introduction}

It is well known that the spherically symmetric linear diffusion equation

$$
\frac{\partial c}{\partial t}=\frac{1}{r^{2}} \frac{\partial}{\partial r}\left(r^{2} \frac{\partial c}{\partial r}\right)
$$

is mapped into the one-dimensional equation

$$
\frac{\partial C}{\partial T}=\frac{\partial^{2} C}{\partial R^{2}}
$$

by the simple discrete local transformation $r=R, t=T, c=C / R$. It has recently been noted (King [12]) that the cylindrically symmetric nonlinear equation

$$
\frac{\partial c}{\partial t}=\frac{1}{r} \frac{\partial}{\partial r}\left(r c^{-1} \frac{\partial c}{\partial r}\right)
$$

is mapped into the one-dimensional equation

$$
\frac{\partial C}{\partial T}=\frac{\partial}{\partial R}\left(C^{-1} \frac{\partial C}{\partial R}\right)
$$

\footnotetext{
'Dept. of Theoretical Mechanics, University of Nottingham, Nottingham, NG7 2RD, U.K.

(C) Copyright Australian Mathematical Society 1992, Serial-fee code 0334-2700/92
} 
by the transformation $r=e^{R}, t=T, c=e^{-2 R} C$. The purpose of this paper is to derive and exploit some generalisations of these transformations.

We start in Section 2 by considering radially symmetric equations of the form

$$
\frac{\partial c}{\partial t}=\frac{1}{r^{N-1}} \frac{\partial}{\partial r}\left(r^{N-1} D(c) \frac{\partial c}{\partial r}\right)
$$

with $D(c)=c^{n}$ and $D(c)=e^{c}$. By straightforward translations and rescalings our results can be extended to diffusivities $D(c)=D_{0}\left(c+c_{0}\right)^{n}$ and $D(c)=D_{0} e^{c / c_{0}}$ where $D_{0}$ and $c_{0}$ are arbitrary constants. We note that the apparently more general equation

$$
\frac{\partial c}{\partial t}=x^{l} \frac{\partial}{\partial x}\left(x^{k} D(c) \frac{\partial c}{\partial x}\right)
$$

discussed by Hill and Hill [10], [8] may be written in the form (1.1) with $N=2(1-l) /(2-(k+l))$ by writing

$$
r=x^{1-(k+l) / 2} /(1-(k+l) / 2) \text {, }
$$

provided that $k+l \neq 2$.

The transformations we derive are applied to relate known exact solutions and to derive new ones. In Section 3 we then extend and apply known Lie group results for multidimensional equations of the form

$$
\partial c / \partial t=\nabla \cdot(D(c) \nabla c)
$$

in a similar fashion.

We conclude with some discussion and mention of some generalisations.

\section{Radially symmetric equations}

\subsection{Introduction}

This section is concerned with the equations

$$
\frac{\partial c}{\partial t}=\frac{1}{r^{N-1}} \frac{\partial}{\partial r}\left(r^{N-1} c^{n} \frac{\partial c}{\partial r}\right)
$$

and

$$
\frac{\partial c}{\partial t}=\frac{1}{r^{N-1}} \frac{\partial}{\partial r}\left(r^{N-1} e^{c} \frac{\partial c}{\partial r}\right) .
$$

Since such equations can arise in contexts in which $N$ does not represent the dimension, we shall consider arbitrary values of $N$.

To simplify the algebra we first introduce

$$
\left.\begin{array}{ll}
x=\left(\frac{r}{|2-N|}\right)^{2-N} & \text { for } N \neq 2, \\
x=\ln r & \text { for } N=2,
\end{array}\right\}
$$


and for (2.1)

$$
\left.\begin{array}{ll}
u=c^{n+1} & \text { for } n \neq-1, \\
u=\ln c & \text { for } n=-1,
\end{array}\right\}
$$

and for (2.2)

$$
u=e^{c}
$$

we define

$$
m=-\frac{n}{n+1}, \quad M=-\frac{2(1-N)}{(2-N)},
$$

with $m=-1$ for (2.2) corresponding to the limit $n \rightarrow \infty$. Then both (2.1) and (2.2) may be written as

$$
\begin{aligned}
& x^{M} u^{m} \frac{\partial u}{\partial t}=\frac{\partial^{2} u}{\partial x^{2}} \quad \text { for } n \neq-1, N \neq 2, \\
& e^{2 x} u^{m} \frac{\partial u}{\partial t}=\frac{\partial^{2} u}{\partial x^{2}} \quad \text { for } n \neq-1, N=2, \\
& x^{M} e^{u} \frac{\partial u}{\partial t}=\frac{\partial^{2} u}{\partial x^{2}} \quad \text { for } n=-1, N \neq 2, \text { and } \\
& e^{2 x} e^{u} \frac{\partial u}{\partial t}=\frac{\partial^{2} u}{\partial x^{2}} \quad \text { for } n=-1, N=2 .
\end{aligned}
$$

Before proceeding to derive new mappings for (2.6)-(2.9), we first note the following well-established continuous transformations under which they are invariant:

(2.6)-(2.9) under $X=x, T=t+\alpha, U=u$;

(2.6) and (2.7) under $X=x, T=\alpha^{m} t, U=\alpha u$;

(2.8) and (2.9) under $X=x, T=e^{\alpha} t, U=u+\alpha$;

(2.6) and (2.8) with $M=0$ under $X=x+\alpha, T=t, U=u$;

(2.6) under $X=\alpha^{-m} x, T=t, U=\alpha^{(M+2)} u$;

(2.7) under $X=x-m \ln \alpha, T=t, U=\alpha^{2} u$;

(2.8) under $X=\alpha x, T=t, U=u-(M+2) \ln \alpha$;

(2.9) under $X=x+\alpha, T=t, U=u-2 \alpha$.

In each case $\alpha$ is an arbitrary constant. In what follows, we shall ignore parameters corresponding to these transformations. 
We also note the following discrete transformations under which (2.6)(2.9) are invariant (in each case $k$ is an arbitrary integer):

(2.6) and (2.7) under $X=x, T=t, U=e^{(2 \pi k / m) i} u$,

(2.8) and (2.9) under $X=x, T=t, U=u+2 \pi k i$,

(2.6) and (2.8) under $X=e^{(2 \pi k(M+2))} x, T=t, U=u$,

(2.7) and (2.8) under $X=x+\pi k i, T=t, U=u$.

These transformations can be derived in the course of the analysis which follows, but since they are given here, we shall not usually refer to them again.

We shall derive our mappings directly, by restricting attention to transformations of the form

$$
X=g(x), \quad T=t, \quad U(X, T)=h(x, u(x, t))
$$

with

$$
h(x, u)=u / f(x) \text { for (2.6) and (2.7), }
$$

and

$$
h(x, u)=u-f(x) \text { for (2.8) and (2.9), }
$$

and we shall determine $f(x)$ and $g(x)$ in order to provide mappings between equations of the form (2.6)-(2.9). These transformations may be continuous or discrete and may map an equation into itself or into another equation of the form (2.6)-(2.9).

The analysis for (2.8) and.(2.9) is simplest, so we start with the case $n=$ -1 .

\section{$2.2 n=-1$}

2.2.1 Derivation of transformations. The appropriate form of (2.18) for $n=-1$ is

$$
X=g(x), \quad T=t, \quad U=u-f(x),
$$

and (2.8), (2.9) then transform respectively to

$$
\begin{aligned}
& x^{M} e^{f} e^{U} \frac{\partial U}{\partial T}=\left(\frac{d g}{d x}\right)^{2} \frac{\partial^{2} U}{\partial X^{2}}+\frac{d^{2} g}{d x^{2}} \frac{\partial U}{\partial X}+\frac{d^{2} f}{d x^{2}}, \text { and } \\
& e^{2 x} e^{f} e^{U} \frac{\partial U}{\partial T}=\left(\frac{d g}{d x}\right)^{2} \frac{\partial^{2} U}{\partial X^{2}}+\frac{d^{2} g}{d x^{2}} \frac{\partial U}{\partial X}+\frac{d^{2} f}{d x^{2}},
\end{aligned}
$$

so we must impose the conditions

$$
\frac{d^{2} f}{d x^{2}}=0, \quad \frac{d^{2} g}{d x^{2}}=0,
$$


giving $f=f_{0}+f_{1} x, g=g_{0}+g_{1} x$, where $f_{0}, f_{1}, g_{0}$ and $g_{1}$ are arbitrary constants. Equation (2.8) then becomes

$$
\left(X-g_{0}\right)^{M} \exp \left(\left\{f_{1} X+f_{0} g_{1}-f_{1} g_{0}\right\} / g_{1}\right) e^{U} \frac{\partial U}{\partial T}=g_{1}^{M+2} \frac{\partial^{2} U}{\partial X^{2}}
$$

while (2.9) maps to

$$
\exp \left(\left\{\left(f_{1}+2\right) X+f_{0} g_{1}-\left(f_{1}+2\right) g_{0}\right\} / g_{1}\right) e^{U} \frac{\partial U}{\partial T}=g_{1}^{2} \frac{\partial^{2} U}{\partial X^{2}} .
$$

In (2.19), if $M \neq 0$ we must set

$$
g_{0}=0, \quad f_{1}=0, \quad f_{0}=(M+2) \ln g_{1},
$$

which corresponds to (2.16) and gives nothing new, though by exploiting the multivaluedness of $\ln g_{1}$ we may also derive the discrete invariants of (2.8) referred to in Section 2.1.

When $M=0$ in (2.19) we may take $f_{1}=2 g_{1}, f_{0}-2 g_{0}=2 \ln g_{1}$, to give a mapping from (2.8) into (2.9). The parameter $f_{0}$ corresponds to (2.16) while $g_{0}$ corresponds to $(2.13)$ so we set

$$
f_{0}=0, \quad g_{0}=0, \quad f_{1}=2, \quad g_{1}=1
$$

to give

$$
X=x, \quad T=t, \quad U=u-2 x .
$$

Hence the transformation

$$
R=e^{r}, \quad T=t, \quad C=e^{-2 r} c
$$

maps

$$
\frac{\partial c}{\partial t}=\frac{\partial}{\partial r}\left(c^{-1} \frac{\partial c}{\partial r}\right)
$$

into

$$
\frac{\partial C}{\partial T}=\frac{1}{R} \frac{\partial}{\partial R}\left(R C^{-1} \frac{\partial C}{\partial R}\right) .
$$

This transformation has already been referred to in Section 1. Turning now to (2.20), by choosing

$$
f_{0}=0, \quad g_{0}=0, \quad f_{1}=-2, \quad g_{1}=1
$$

we have a mapping from (2.9) into (2.8) with $M=0$ taking the form

$$
X=x, \quad T=t, \quad U=u+2 x .
$$

Hence

$$
R=\ln r, \quad T=t, \quad C=r^{2} c
$$


maps

$$
\frac{\partial c}{\partial t}=\frac{1}{r} \frac{\partial}{\partial r}\left(r c^{-1} \frac{\partial c}{\partial r}\right)
$$

into

$$
\frac{\partial C}{\partial T}=\frac{\partial}{\partial R}\left(C^{-1} \frac{\partial C}{\partial R}\right) .
$$

This is the inverse of the mapping (2.21) and will be exploited shortly. Choosing

$$
f_{1}+2=2 g_{1}, \quad f_{0}-2 g_{0}=2 \ln g,
$$

in (2.20) we have a mapping of (2.9) into itself. The parameter $g_{0}$ corresponds to (2.17) and we set

$$
g_{0}=0, \quad g_{1}=1+\beta, \quad f_{0}=2 \ln (1+\beta), \quad f_{1}=2 \beta,
$$

where $\beta$ is an arbitrary constant. Hence

$$
X=(1+\beta) x, \quad T=t, \quad U=u-2 \beta x-2 \ln (1+\beta)
$$

so that

$$
R=r^{1+\beta}, \quad T=t, \quad C=(1+\beta)^{-2} r^{-2 \beta} c
$$

maps (2.23) into itself.

The transformations we have derived for (2.8) and (2.9) could be spotted without the analysis given here, but the value of the systematic approach will become more evident in dealing with (2.6).

Nariboli [15] went some way towards giving a Lie group analysis for an equation equivalent to (1.1) with $N=2$ and $N=3$ (as well as $N=1$ ), but does not give a result corresponding to the continuous transformation (2.26). Mapping (2.9) into (2.8) with $M=0$ maps invariance properties of the former into those of the latter as follows: $(2.10)$ goes to $(2.10),(2.12)$ to (2.12), (2.17) to (2.13), and (2.25) to (2.8) (with $\alpha=1+\beta$ ).

2.2.2 Applications of transformations. The transformations that we have derived here have already been used to derive some exact solutions in King [12], but some further results may also be noted.

We start by listing the similarity solutions of $(2.24)$ together with the solutions of (2.23) to which they map; in each case $\gamma$ is an arbitrary constant.

$$
\begin{aligned}
\text { (i) } & C=F(R-\gamma T), & c & =e^{-2 \gamma t} f\left(r / e^{\gamma t}\right) ; \\
\text { (ii) } & C=T^{1-2 \gamma} F\left(R / T^{\gamma}\right), & c & =r^{-2} t^{1-2 \gamma} F\left(\ln r / t^{\gamma}\right) ; \\
\text { (iii) } & C=e^{-2 \gamma T} F\left(R / e^{\gamma T}\right), & c & =r^{-2} e^{-2 \gamma t} F\left(\ln r / e^{\gamma t}\right) ; \\
\text { (iv) } & C=T F(R+\gamma \ln T), & c & =t^{1-2 \gamma} f\left(r / t^{\gamma}\right) ;
\end{aligned}
$$


where in (i) and (iv) $f(\eta)=\eta^{-2} F(\ln \eta)$. It is evident from (2.26) also that solutions to (2.23) of the form (2.28) for all values of $\gamma \neq 0$ can be derived from the solution for a single $\gamma$.

Further exact solutions to (2.23) of the form (2.27) not noted in King [12] are the general solution of the form $c=r^{-2} F\left(\ln r / t^{\frac{1}{2}}\right)$ corresponding to taking $\gamma=\frac{1}{2}$ in (2.27), which can be derived from the Fujita [6] solution to (2.24), and the separable solution $c=t r^{-2} F(\ln r)$ which corresponds to $\gamma=0$. In the latter case we have

$$
\frac{d}{d R}\left(F^{-1} \frac{d F}{d R}\right)=F
$$

with general solution

$$
F(R)=F_{0} / \sinh ^{2}\left\{\left(\frac{F_{0}}{2}\right)^{\frac{1}{2}}\left(R+R_{0}\right)\right\}
$$

where $F_{0}$ and $R_{0}$ are arbitrary constants. We note that if we write the separable solutions to (2.24) and (2.23) in the forms

$$
C=T e^{\Psi(R)}, \quad c=t e^{\psi(r)}
$$

we have

$$
\frac{d^{2} \Psi}{d R^{2}}=e^{\Psi}, \quad \frac{1}{r} \frac{d}{d r}\left(r \frac{d \psi}{d r}\right)=e^{\psi},
$$

and the transformation (2.22) reads

$$
R=\ln r, \quad \Psi=\psi+2 \ln r,
$$

giving the usual transformation between the one-dimensional and cylindrically symmetric Liouville equations.

$2.3 n=-1$

2.3.1 Derivation of transformations. We now turn to (2.6) and (2.7), the required form of $(2.18)$ then being

$$
X=g(x), \quad T=t, \quad U=u / f(x) .
$$

Under this transformation (2.6) and (2.7) become, respectively,

$$
x^{M} f^{m+1} U^{m} \frac{\partial U}{\partial T}=f\left(\frac{d g}{d x}\right)^{2} \frac{\partial^{2} U}{\partial X^{2}}+\left(2 \frac{d f}{d x} \frac{d g}{d x}+f \frac{d^{2} g}{d x^{2}}\right) \frac{\partial U}{\partial X}+\frac{d^{2} f}{d x^{2}} U,
$$

and

$$
e^{2 x} f^{m+1} U^{m} \frac{\partial U}{\partial T}=f\left(\frac{d g}{d x}\right)^{2} \frac{\partial^{2} U}{\partial X^{2}}+\left(2 \frac{d f}{d x} \frac{d g}{d x}+f \frac{d^{2} g}{d x^{2}}\right) \frac{\partial U}{\partial X}+\frac{d^{2} f}{d x^{2}} U
$$


Hence we must impose conditions

$$
\frac{d^{2} f}{d x^{2}}=0, \quad 2 \frac{d f}{d x} \frac{d g}{d x}+f \frac{d^{2} g}{d x^{2}}=0,
$$

so that $f=f_{0}+f_{1} x$ where $f_{0}$ and $f_{1}$ are arbitrary constants. If $f_{1}=$ 0 then $g=g_{0}+g_{1} x$ where $g_{0}$ and $g_{1}$ are arbitrary constants, and this transformation is simply a rescaling of $u$ and a translation and rescaling of $x$. We may therefore limit discussion to the case $f_{1} \neq 0$ when $g=$ $g_{0}+g_{1} /\left(f_{0}+f_{1} x\right)$. Writing $g_{0}=-F_{0} / F_{1}, g_{1}=1 / F_{1}$, gives

$$
g=\frac{1}{F_{1}}\left(\frac{1}{f_{0}+f_{1} x}-F_{0}\right),
$$

so that $\left(F_{0}+F_{1} X\right)\left(f_{0}+f_{1} x\right)=1$. Equations (2.29) and (2.30) now read, respectively,

$$
\left(F_{0}+F_{1} X\right)^{-(m+M+4)}\left(1-f_{0} F_{0}-f_{0} F_{1} X\right)^{M} U^{m} \frac{\partial U}{\partial T}=\frac{f_{1}^{M+2}}{F_{1}^{2}} \frac{\partial^{2} U}{\partial X^{2}}
$$

and

$$
\left(F_{0}+F_{1} X\right)^{-(m+4)} \exp \left(\frac{2}{f_{1}}\left(\frac{1}{F_{0}+F_{1} X}-f_{0}\right)\right) U^{m} \frac{\partial U}{\partial T}=\frac{f_{1}^{2}}{F_{1}^{2}} \frac{\partial^{2} U}{\partial X^{2}} .
$$

Since $F_{1} \neq 0,(2.32)$ cannot be written in the form (2.6)-(2.9) so we obtain no new results for (2.7). For (2.6), however, there are a variety of possibilities; (2.31) becomes an equation of the form (2.6) in all the following five cases:

(i) $M=0, \quad F_{0}=0$.

Imposing $F_{1}^{m+2} f_{1}^{2}=1,(2.31)$ then reads

$$
X^{-(m+4)} U^{m} \frac{\partial U}{\partial T}=\frac{\partial^{2} U}{\partial X^{2}}
$$

The parameter $f_{0}$ corresponds to $(2.13)$ and $f_{1}$ to $(2.14)$ so taking

$$
f_{0}=0, \quad f_{1}=1, \quad F_{0}=0, \quad F_{1}=1
$$

the transformation is

$$
X=1 / x, \quad T=t, \quad U=u / x .
$$

(ii) $m+M+4=0, \quad f_{0}=0$.

Imposing $F_{1}^{2}=f_{1}^{M+2},(2.31)$ now reads

$$
U^{m} \frac{\partial U}{\partial T}=\frac{\partial^{2} U}{\partial X^{2}}
$$


$F_{0}$ corresponds to the invariance of (2.33) under translations of $X$, and $f_{1}$ corresponds to (2.14) so taking

$$
f_{0}=0, \quad f_{1}=1, \quad F_{0}=0, \quad F_{1}=1
$$

yields

$$
X=1 / x, \quad T=t, \quad U=u / x .
$$

This is the inverse of the transformation given in (i). The condition $m+M+4=0$ is equivalent to

$$
n=-2(N-3) /(N-4)
$$

and the transformation (2.34) becomes for $N \neq 4$

$$
R=\left(\frac{r}{|N-2|}\right)^{N-2}, \quad T=t, \quad C=\left(\frac{r}{|N-2|}\right)^{-(N-4)} c,
$$

which maps $(2.1)$ into

$$
\frac{\partial C}{\partial T}=\frac{\partial}{\partial R}\left(C^{-\frac{2(N-3)}{(N-4)}} \frac{\partial C}{\partial R}\right)
$$

when the condition (2.35) holds. Thus when $n=-2(N-3) /(N-4)$, the radially symmetric equation (2.1) maps to its one-dimensional equivalent. For $N=3$ we have $n=0$, giving the usual reduction for linear diffusion. As $N \rightarrow 2$ we have $n \rightarrow-1$ as in Section 2.2. For $N=1$ we have $n=-4 / 3$ and this case is discussed in more detail below. Finally, for $N=4$ we have a transformation from

$$
\frac{\partial c}{\partial t}=\frac{1}{r^{3}} \frac{\partial}{\partial r}\left(r^{3} e^{c} \frac{\partial c}{\partial r}\right)
$$

to

$$
\frac{\partial C}{\partial T}=\frac{\partial}{\partial R}\left(e^{C} \frac{\partial C}{\partial R}\right)
$$

given by

$$
R=r^{2} / 4, \quad T=t, \quad C=c+2 \ln (r / 2) .
$$

(iii) $f_{0}=0, \quad F_{0}=0$.

Imposing $F_{1}^{m+M+2} f_{1}^{M+2}=1,(2.31)$ now reads

$$
X^{-(m+M+4)} U^{m} \frac{\partial U}{\partial T}=\frac{\partial^{2} U}{\partial X^{2}} .
$$

Taking

$$
f_{0}=0, \quad f_{1}=1, \quad F_{0}=0, \quad F_{1}=1
$$

we have

$$
X=1 / x, \quad T=t, \quad U=u / x,
$$


giving a transformation between (2.6) and (2.41). Equation (2.1) with $n \neq-2 / N$ is mapped into

$$
\frac{\partial C}{\partial T}=\frac{1}{R^{N^{\prime}-1}} \frac{\partial}{\partial R}\left(R^{N^{\prime}-1} C^{n} \frac{\partial C}{\partial R}\right)
$$

where

$$
N^{\prime}=\frac{2(2 n-N+4)}{(n N+2)}, \quad N=\frac{2\left(2 n-N^{\prime}+4\right)}{\left(n N^{\prime}+2\right)},
$$

by the transformation

$$
\begin{gathered}
R=\left|\frac{2(n+1)(N-2)}{n N+2}\right|\left(\frac{r}{|N-2|}\right)^{\frac{n N+2}{2(n+1)}}, \quad T=t, \\
C=\left(\frac{r}{|N-2|}\right)^{\frac{N-2}{n+1}} c .
\end{gathered}
$$

The transformation (2.36) is a special case of (2.44) corresponding to $N^{\prime}=1$. Similarly, equation (2.2) is mapped into

$$
\frac{\partial C}{\partial T}=\frac{1}{R^{(4 / N)-1}} \frac{\partial}{\partial R}\left(R^{(4 / N)-1} e^{C} \frac{\partial C}{\partial R}\right)
$$

by

$R=\left|\frac{2(N-2)}{N}\right|\left(\frac{r}{|N-2|}\right)^{\frac{N}{2}}, \quad T=t, \quad C=c+(N-2) \ln \left(\frac{r}{|N-2|}\right)$.

The transformation (2.45) generalises (2.40).

From (2.43) we see that we have a mapping from $N=3$ to $N^{\prime}=1$ (or vice versa) if $n=0$, from $N=1$ to $N^{\prime}=1$ if $n=-4 / 3$, and from $N=3$ to $N^{\prime}=3$ if $n=-4 / 5$. The transformation gives a discrete mapping of (2.1) into itself when $N^{\prime}=N$ so that $n=-4 /(N+2)$; in this case (2.44) reads

$$
R=|N-2|^{2} r^{-1}, \quad T=t, \quad C=(r /|N-2|)^{N+2} c .
$$

(iv) $M=0, \quad m=-4$.

This implies $N=1$ and $n=-4 / 3$ and imposing $f_{1}^{2}=F_{1}^{2},(2.31)$ reads

$$
U^{4} \frac{\partial U}{\partial T}=\frac{\partial^{2} U}{\partial R^{2}}
$$

so that we have mapped (2.8) into itself. The parameters $f_{0}$ and $F_{0}$ are arbitrary, corresponding respectively to translations of $x$ and $X$. Choosing

$$
f_{0}=1, \quad f_{1}=\beta, \quad F_{0}=1, \quad F_{1}=-\beta,
$$


where $\beta$ is an arbitrary constant, gives the continuous transformation

$$
X=\frac{x}{1+\beta x}, \quad T=t, \quad U=\frac{u}{1+\beta x},
$$

so that the transformation

$$
R=\frac{r}{1+\beta r}, \quad T=t, \quad C=(1+\beta r)^{3} c
$$

maps

$$
\frac{\partial c}{\partial t}=\frac{\partial}{\partial r}\left(c^{-\frac{4}{3}} \frac{\partial c}{\partial r}\right)
$$

into itself.

It is well known that (2.49) possesses one more group invariant than (2.1) for $N=1$ with $n \neq-4 / 3$ and the form of the infinitesimals corresponding to (2.48) has been established by Lie group methods (see, for example, Ovsiannikov [16] and Nariboli [15]). The global form of the invariant expressed by (2.48) may alternatively be derived from the infinitesimals, and is exploited below.

(v) $m+M+4=0, \quad f_{0} F_{0}=1$.

Then we impose $f_{0}^{M}\left(-F_{1} / f_{1}\right)^{M+2}=1$ and (2.31) becomes

$$
X^{M} U^{m} \frac{\partial U}{\partial T}=\frac{\partial^{2} U}{\partial X^{2}}
$$

so that (2.6) is mapped into itself. Choosing

$$
f_{0}=1, \quad f_{1}=\beta, \quad F_{0}=1, \quad F_{1}=-\beta,
$$

where $\beta$ is again an arbitrary constant, gives

$$
X=\frac{x}{1+\beta x}, \quad T=t, \quad U=\frac{u}{1+\beta x},
$$

which is a continuous mapping of (2.6) into itself. The case $m+M+4=0$, giving $n=-2(N-3) /(N-4)$, also arises in (ii) above where a transformation to (2.33) corresponding to $M=0$ is given. The invariance properties of (2.6) with $m+M+4=0$ map under (2.34) to those of (2.6) with $M=0$ as follows: (2.10) goes to (2.10), (2.11) goes to (2.11), (2.14) goes to (2.14), and (2.50) goes to (2.13) (with $\alpha=\beta$ ). Equation (2.50) leads to the following continuous mapping of $(2.1)$ with $n=-2(N-3) /(N-4)$ into itself:

$$
R=\left(r^{N-2}+\gamma\right)^{\frac{1}{N-2}}, \quad T=t, \quad C=\left(1+\gamma r^{-(N-2)}\right)^{\frac{N-4}{N-2}} c,
$$

where

$$
\gamma=\beta|N-2|^{N-2} .
$$

In addition, (2.2) with $N=4$ is mapped into itself by

$$
R=\left(r^{2}+4 \beta\right)^{\frac{1}{2}}, \quad T=t, \quad C=c-\ln \left(1+4 \beta / r^{2}\right) .
$$


2.3.2 Summary. We have derived the following for $N \neq 2, n \neq-1$;

(I) Discrete transformations

(a) Equation (2.1) is mapped to

$$
\frac{\partial C}{\partial T}=\frac{1}{R^{N^{\prime}-1}} \frac{\partial}{\partial R}\left(R^{N^{\prime}-1} C^{n} \frac{\partial C}{\partial R}\right),
$$

where $N^{\prime}=2(2 n-N+4) /(n N+2)$ by,

$$
\begin{gathered}
R=\left|\frac{2(n+1)(N-2)}{n N+2}\right|\left(\frac{r}{|N-2|}\right)^{\frac{n N+2}{2(n+1)}}, \quad T=t, \\
C=\left(\frac{r}{|N-2|}\right)^{\frac{N-2}{n+1}} c ;
\end{gathered}
$$

see (2.42)-(2.44). When $n=-2(N-3) /(N-4)$ we have $N^{\prime}=1$; see (2.35)-(2.37). When $n=-4 /(N+2)$ we have $N^{\prime}=N$; see (2.46).

(b) Equation (2.2) is mapped to

$$
\frac{\partial C}{\partial T}=\frac{1}{R^{(4 / N)-1}} \frac{\partial}{\partial R}\left(R^{(4 / N)-1} e^{C} \frac{\partial C}{\partial R}\right)
$$

by

$$
\begin{gathered}
R=\left|\frac{2(N-2)}{N}\right|\left(\frac{r}{|N-2|}\right)^{\frac{N}{2}}, \quad T=t, \quad C=c+(N-2) \ln \left(\frac{r}{|N-2|}\right) ; \\
\text { see (2.45). When } N=4,(2.53) \text { becomes }(2.39) \text {; see }(2.38)-(2.40) \\
\text { When } N=-2,(2.2) \text { is mapped into itself. }
\end{gathered}
$$

(II) Continuous transformations

(a) When $n=-2(N-3) /(N-4),(2.1)$ is mapped to itself by

$$
R=\left(r^{N-2}+\gamma\right)^{\frac{1}{N-2}}, \quad T=t, \quad C=\left(1+\gamma r^{-(N-2)}\right)^{\frac{N-4}{N-2}} c
$$

see (2.51). For the specialisation of this result for $N=1, n=-4 / 3$, see $(2.48)-(2.49)$.

(b) When $N=4,(2.2)$ is mapped into itself by

$$
R=\left(r^{2}+\gamma\right)^{\frac{1}{2}}, \quad T=t, \quad C=c-\ln \left(1+\gamma / r^{2}\right) ;
$$

see (2.52).

2.3.3 Application of transformations. Many exact solutions to (2.1) are already known (see, for example, Lacey et al. [14], Hill [10], Hill and Hill [8] and King [12]) and our transformations may be used to derive new solutions from these. 
In particular, (2.1) and (2.42) have the following classes of similarity solution which are mapped into one another by (2.44):

(i) $c=t^{-a} f\left(r / t^{\frac{1}{2}(1-n a)}\right), \quad C=T^{-A} F\left(R / T^{\frac{1}{2}(1-n A)}\right)$,

where $a$ is an arbitrary constant and

$$
A=\frac{(n N+2) a-(N-2)}{2(n+1)}=\frac{2(n+1) a+\left(N^{\prime}-2\right)}{\left(n N^{\prime}+2\right)} .
$$

(ii) $c=e^{-a t} f\left(r e^{\frac{1}{2} n a t}\right)$,

$$
C=e^{-A T} F\left(R e^{\frac{1}{2} n A T}\right),
$$

where

$$
A=\frac{(n N+2) a}{2(n+1)}=\frac{2(n+1) a}{\left(n N^{\prime}+2\right)} .
$$

Closed-form solutions of the type (2.54) are known for $a=N /(n N+2)$ (the instantaneous source solution) and $a=1 /(n+1)$ (the 'dipole' solution); see King [12] and references therein. These map respectively to solutions with $A=1 /(n+1)$ and $A=N^{\prime} /\left(n N^{\prime}+2\right)$ and therefore give little new. We note, however, that in King [12] the general similarity solution of 'dipole' form (i.e. for $a=1 /(n+1)$ ) was constructed for $n=\frac{1}{2} N-2$ and this maps to the similarity solution $C=F\left(R / T^{\frac{1}{2}}\right)$ of $(2.42)$ with $N^{\prime}=0$. This solution is also easily constructed directly; writing $Y=R / T^{\frac{1}{2}}$ gives

$$
-\frac{1}{2} Y \frac{d F}{d Y}=Y \frac{d}{d Y}\left(Y^{-1} F^{n} \frac{d F}{d Y}\right)
$$

so that

$$
\int_{F_{1}}^{F} \frac{F^{n}}{F-F_{0}} D F=-\frac{1}{4} Y^{2}
$$

where $F_{0}$ and $F_{1}$ are arbitrary constants.

Further progress is possible in the special case $n=-2(N-3) /(N-4)$ when (2.36) maps (2.1) to (2.37) and the continuous transformation (2.51) mapping (2.1) into itself exists.

From (2.51) it follows that if $c=g(r, t)$ satisfies

$$
\frac{\partial c}{\partial t}=\frac{1}{r^{N-1}} \frac{\partial}{\partial r}\left(r^{N-1} c^{-\frac{2(N-3)}{(N-4)}} \frac{\partial c}{\partial r}\right)
$$

then so does

$$
c=\left(1+\gamma r^{-(N-2)}\right)^{-\left(\frac{N-4}{N-2}\right)} g\left(\left(r^{N-2}+\gamma\right)^{\frac{1}{N-2}}, t\right)
$$

for any value of $\gamma$. In particular, we may generalise the similarity solutions 
listed above as follows:

(i) $c=t^{-a} f\left(r / t^{\frac{1}{2}+\left(\frac{N-3}{N-4}\right) a}\right)$

maps to

$$
c=t^{-a}\left(1+\gamma r^{-(N-2)}\right)^{-\left(\frac{N-4}{N-2}\right)} f\left(\left(r^{N-2}+\gamma\right)^{\frac{1}{N-2}} / t^{\frac{1}{2}+\left(\frac{N-3}{N-4}\right) a}\right) .
$$

Similarity solutions equivalent to this form were discussed by Hill [10] for the case $N=1, n=-4 / 3$; the fact that they could be derived directly from the standard similarity forms (2.56) was not noted however.

(ii) $c=e^{-a t} f\left(r / \exp \left(\left(\frac{N-3}{N-4}\right) a t\right)\right)$

maps to

$$
c=e^{-a t}\left(1+\gamma r^{-(N-2)}\right)^{-\left(\frac{N-4}{N-2}\right)} f\left(\left(r^{N-2}+\gamma\right)^{\frac{1}{N-2}} / \exp \left(\left(\frac{N-3}{N-4}\right) a t\right)\right) .
$$

Closed-form solutions to (2.55) are given by the instantaneous source solution

$$
c=\left\{-\frac{(N-3)}{2(N-2)^{2}}\left(\mu t^{\left.\left.-\frac{N(N-3)}{(N-2)^{2}}+r^{2} t^{-1}\right)\right\}^{-\frac{(N-4)}{2(N-3)}}}\right.\right.
$$

and the 'dipole' solution

$$
c=r^{N-4}\left\{-\frac{(N-3)}{2(N-2)^{2}}\left(\mu t^{-(N-3)}+r^{2(N-2)} t^{-1}\right)\right\}^{-\frac{(N-4)}{2(N-3)}},
$$

where in each case $\mu$ is an arbitrary constant. Using (2.51) these map to

$$
\begin{aligned}
c= & r^{N-4}\left(r^{N-2}+\gamma\right)^{-\left(\frac{N-4}{N-2}\right)} \\
& \times\left\{-\frac{(N-3)}{2(N-2)^{2}}\left(\mu t^{-\frac{N(N-3)}{(N-2)^{2}}}+\left(r^{N-2}+\gamma\right)^{\frac{2}{N-2}} t^{-1}\right)\right\}^{-\frac{(N-4)}{2(N-3)}}
\end{aligned}
$$

and

$$
c=r^{N-4}\left\{-\frac{(N-3)}{2(N-2)^{2}}\left(\mu t^{-(N-3)}+\left(r^{N-2}+\gamma\right)^{2} t^{-1}\right)\right\}^{-\frac{(N-4)}{2(N-3)}}
$$

respectively. When $N=1$ these two solutions are equivalent and correspond to the 'source-dipole' solution derived by Hill [10], [8].

We may also exploit the reduction to (2.37) to derive new solutions. The instantaneous source and dipole solutions of (2.37) lead (using the invariance of (2.37) under translations of $R$ ) respectively to (2.58) and (2.57). Equation (2.37) has additional similarity solutions not shared by (2.1) with $N \neq 1$ as follows:

(iii) $C=F(R-q T)$, where $q$ is arbitrary constant. 
These are the travelling waves and map under (2.36) to solutions of (2.55) of the form

$$
c=\left(\frac{r}{|N-2|}\right)^{N-4} F\left(\left(\frac{r}{|N-2|}\right)^{N-2}-q T\right) .
$$

$F$ can be determined exactly in the form

$$
\int_{F_{1}}^{F} \frac{F^{-2(N-3) /(N-4)}}{F-F_{0}} d F=-q(R-q T),
$$

where $F_{0}$ and $F_{1}$ are arbitrary constants. Taking $F_{0}=F_{1}=0$ gives

$$
C=\left\{\frac{2(N-3)}{(N-4)} q(R-q T)\right\}^{-\frac{(N-4)}{2(N-3)}}
$$

and this leads to

$$
c=r^{N-4}\left\{\frac{2(N-3)}{(N-4)(N-2)^{2}} \beta\left(r^{N-2}-\beta t\right)\right\}^{-\frac{(N-4)}{2(N-3)}}
$$

(where $\beta=q|N-2|^{N-2}$ ) as a solution to (2.55). This solution may also be written in the form (2.56) with $a=-(N-4)^{2} / 2(N-2)(N-3)$.

(iv) $C=T^{\frac{(N-3)}{2(N-2)}} F(R+\mu \ln T)$, where $\mu$ is an arbitrary constant.

This maps to solutions of (2.55) of the form

$$
c=t^{\frac{(N-3)}{2(N-2)}}\left(\frac{r}{|N-2|}\right)^{N-4} F\left(\left(\frac{r}{|N-2|}\right)^{N-2}+\mu \ln t\right) .
$$

When $\mu=0$ we have the separable solutions to both (2.37) and (2.55). The former is easily expressed in terms of quadratures (see, for example, Hill [10]) and may be used to deduce the latter.

We note that all our additional similarity forms (as well as the oneparameter group represented by (2.51)) can also be derived by standard Lie group methods. When $N=1$, so $n=-4 / 3,(2.36)$ reads

$$
R=r^{-1}, \quad T=t, \quad C=r^{3} c
$$

and gives a discrete transformation of (2.49) into itself. This case also possesses the continuous transformation (2.48) which is a special case of (2.51) 
and this leads to further additional similarity forms as follows:

(iii) $c=f(r-q t)$

maps to

$$
c=(1+\beta r)^{-3} f\left(\frac{r}{1+\beta r}-q t\right) .
$$

(iv) $c=t^{\frac{3}{4}} f(r+\mu \ln t)$

maps to

$$
c=t^{\frac{3}{4}}(1+\beta r)^{-3} f\left(\frac{r}{1+\beta r}+\mu \ln t\right) .
$$

In particular, a solution of the form (2.59),

$$
c=\left(\frac{4}{3} q(r-q t)\right)^{-\frac{3}{4}}
$$

gives

$$
c=(1+\beta r)^{-3}\left(\frac{4}{3} q\left(\frac{r}{1+\beta r}-q t\right)\right)^{-\frac{3}{4}}
$$

as a further solution to (2.49).

\section{Multidimensional diffusion}

\subsection{Introduction}

In this section we shall exploit and extend known Lie group results for equations of the form

$$
\frac{\partial c}{\partial t}=\nabla \cdot\left(c^{n} \nabla c\right)
$$

in both two and three dimensions.

Lie group methods are well established for nonlinear diffusion equations. The one-dimensional problem was first considered by Ovsiannikov [16] and later by Nariboli [15], Ames [1], Bluman and Cole [3] and Hill [9]. Higher dimensional problems have been studied by Nariboli [15], Branson and Steeb [2], Dorodnitsyn et al. [5] (see also Galaktionov et al. [7]), Kersten and Gragert [11] and Yang et al. [18]. These references show that in $N$ dimensions the invariance group of (3.1) possesses extra parameters when $n=-4 /(N+2)$; as we have already seen, when restricted to the radially symmetric equation, the extra invariant for this case with $N>2$ turns out to be a discrete one. In the special case $N=2, n=-1$ the group becomes infinite-dimensional, as first noted by Nariboli [15].

We shall limit discussion to this two-dimensional case when

$$
\frac{\partial c}{\partial t}=\frac{\partial}{\partial x}\left(c^{-1} \frac{\partial c}{\partial x}\right)+\frac{\partial}{\partial y}\left(c^{-1} \frac{\partial c}{\partial y}\right)
$$


and to the three-dimensional case

$$
\frac{\partial c}{\partial t}=\frac{\partial}{\partial x}\left(c^{-\frac{4}{3}} \frac{\partial c}{\partial x}\right)+\frac{\partial}{\partial y}\left(c^{-\frac{4}{3}} \frac{\partial c}{\partial y}\right)+\frac{\partial}{\partial z}\left(c^{-\frac{4}{3}} \frac{\partial c}{\partial z}\right) .
$$

3.2. $c_{t}=\left(c^{-1} c_{x}\right)_{x}+\left(c^{-1} c_{y}\right)_{y}$

Considering now (3.2), the infinite-dimensional group can be determined directly by seeking a transformation

$$
\begin{gathered}
X=X(x, y), \quad Y=Y(x, y), \quad T=t, \\
C(X, Y, T)=c(x, y, t) / f(x, y)
\end{gathered}
$$

to give

$$
\begin{aligned}
f \frac{\partial C}{\partial T}= & \left\{\left(\frac{\partial X}{\partial x}\right)^{2}+\left(\frac{\partial X}{\partial y}\right)^{2}\right\} \frac{\partial}{\partial X}\left(C^{-1} \frac{\partial C}{\partial X}\right) \\
& +\left\{\left(\frac{\partial Y}{\partial x}\right)^{2}+\left(\frac{\partial Y}{\partial y}\right)^{2}\right\} \frac{\partial}{\partial Y}\left(C^{-1} \frac{\partial C}{\partial Y}\right) \\
& +2\left\{\frac{\partial X}{\partial x} \frac{\partial Y}{\partial x}+\frac{\partial X}{\partial y} \frac{\partial Y}{\partial y}\right\} \frac{\partial^{2}}{\partial X \partial Y}(\ln C) \\
& +\left\{\frac{\partial^{2} X}{\partial x^{2}}+\frac{\partial^{2} X}{\partial y^{2}}\right\} C^{-1} \frac{\partial C}{\partial X}+\left\{\frac{\partial^{2} Y}{\partial x^{2}}+\frac{\partial^{2} Y}{\partial y^{2}}\right\} C^{-1} \frac{\partial C}{\partial Y} \\
& +\left(\frac{\partial^{2}}{\partial x^{2}}(\ln f)+\frac{\partial^{2}}{\partial y^{2}}(\ln f)\right),
\end{aligned}
$$

which gives a mapping of (3.2) into itself provided that

$$
\begin{gathered}
\left(\frac{\partial X}{\partial x}\right)^{2}+\left(\frac{\partial X}{\partial y}\right)^{2}=\left(\frac{\partial Y}{\partial x}\right)^{2}+\left(\frac{\partial Y}{\partial y}\right)^{2}=f \\
\frac{\partial X}{\partial x} \frac{\partial Y}{\partial x}+\frac{\partial X}{\partial y} \frac{\partial Y}{\partial y}=0 \\
\frac{\partial^{2} X}{\partial x^{2}}+\frac{\partial^{2} X}{\partial y^{2}}=\frac{\partial^{2} Y}{\partial x^{2}}+\frac{\partial^{2} Y}{\partial y^{2}}=\frac{\partial^{2}}{\partial x^{2}}(\ln f)+\frac{\partial^{2}}{\partial y^{2}}(\ln f)=0 .
\end{gathered}
$$

This implies that, without loss of generality,

$$
\frac{\partial X}{\partial x}=\frac{\partial Y}{\partial y}, \quad \frac{\partial X}{\partial y}=-\frac{\partial Y}{\partial x}
$$

so that $X$ and $Y$ are harmonic conjugates, and

$$
f=\left(\frac{\partial X}{\partial x}\right)^{2}+\left(\frac{\partial X}{\partial y}\right)^{2} \text {. }
$$


Writing $z=x+i y, Z(z)=X+i Y$ it follows that

$$
f=\left|\frac{d Z}{d z}\right|^{2}
$$

so that

$$
\ln f=\operatorname{Re}\left(2 \ln \frac{d Z}{d z}\right)
$$

and the condition

$$
\frac{\partial^{2}}{\partial x^{2}}(\ln f)+\frac{\partial^{2}}{\partial y^{2}}(\ln f)=0
$$

is automatically satisfied.

The transformation (3.4) thus reads

$$
\begin{gathered}
X=X(x, y), \quad Y=Y(x, y), \quad T=t, \\
c(x, y, t)=\left\{\left(\frac{\partial X}{\partial x}\right)^{2}+\left(\frac{\partial X}{\partial y}\right)^{2}\right\} C(X, Y, T)
\end{gathered}
$$

where $X$ and $Y$ satisfy (3.5) but are otherwise arbitrary.

This transformation may be of value numerically in mapping a problem for (3.2) on a complicated region to one on a computationally more convenient one.

When $X$ and $Y$ are chosen such that the transformation is a continuous one, the infinitesimals corresponding to (3.6) are equivalent to those given in Nariboli [15] and Dorodnitsyn et al [5]. The transformation (3.6) can also be a discrete one. As an example of the latter we note, after first defining

$$
x=r \cos \theta, \quad y=r \sin \theta, \quad X=R \cos \theta \quad Y=R \sin \theta,
$$

that writing $Z=\ln z$ gives

$$
X=\ln r, \quad Y=\theta, \quad T=t, \quad C=r^{2} c,
$$

and this is a generalisation of the discrete transformation (2.22) between cylindrically symmetric and one-dimensional versions of (3.2). As an example of a continuous transformation we write $Z=z^{1+\beta}$ to give

$$
R=r^{1+\beta}, \quad \boldsymbol{\theta}=(1+\beta) \theta, \quad T=t, \quad C=\left(1+\beta^{-2}\right) r^{-2 \beta} c,
$$

which generalises $(2.26)$.

The transformation (3.6) enables many new solutions to (3.2) to be constructed. If $c=g(x, t)$ is any solution to

$$
\frac{\partial c}{\partial t}=\frac{\partial}{\partial x}\left(c^{-1} \frac{\partial c}{\partial x}\right)
$$


it follows from (3.6) that

$$
c=\left\{\left(\frac{\partial X}{\partial x}\right)^{2}+\left(\frac{\partial X}{\partial y}\right)^{2}\right\} g(X(x, y), t)
$$

satisfies (3.2) for any harmonic function $X(x, y)$. The question of determining whether given initial data for a Cauchy problem for (3.2) leads to a solution in the essentially one-dimensional form (3.8) is addressed in the Appendix. Here we exploit known similarity solutions of (3.7) as follows:

(i) $c=f(x-q t)$, where $q$ is an arbitrary constant. Possible forms are

$$
c=f_{0} /\left(1-e^{-q f_{0}(x-q t)}\right)
$$

where $f_{0}$ is an arbitrary constant, and

$$
c=1 / q(x-q t),
$$

corresponding to $f_{0}=0$. These give two-dimensional solutions

$$
c=\frac{\left\{\left(\frac{\partial X}{\partial x}\right)^{2}+\left(\frac{\partial X}{\partial y}\right)^{2}\right\} f_{0}}{1-e^{-q f_{0}(X-q t)}} \text { and } c=\frac{\left\{\left(\frac{\partial X}{\partial x}\right)^{2}+\left(\frac{\partial X}{\partial y}\right)^{2}\right\}}{q(X-q t)} .
$$

Choosing $Z=z^{1+\beta}$ the latter solution becomes

$$
c=\frac{(1+\beta)^{2} r^{2 \beta}}{q\left(r^{1+\beta} \cos (1+\beta) \theta-q t\right)},
$$

which may also be written as a similarity solution of the form

$$
c=t^{-\frac{1-\beta}{1+\beta}} h\left(\frac{x}{t^{\frac{1}{1+\beta}}}, \frac{y}{t^{\frac{1}{1+\beta}}}\right) \text {. }
$$

(ii) $c=t f(x)$.

This solution was given in Section 2.2.2 and takes the form

$$
c=\frac{f_{0} t}{\sinh ^{2}\left\{\left(\frac{f_{0}}{2}\right)^{\frac{1}{2}}\left(x+x_{0}\right)\right\}}
$$

where $f_{0}$ and $x_{0}$ are arbitrary constants. Hence

$$
c=\frac{2\left\{\left(\frac{\partial X}{\partial x}\right)^{2}+\left(\frac{\partial X}{\partial y}\right)^{2}\right\} t}{\sinh ^{2} X}
$$

satisfies (3.2) for any harmonic $X(x, y)$. We note that writing

$$
c=t e^{\psi(x, y)},
$$


(3.2) becomes

$$
\frac{\partial^{2} \psi}{\partial x^{2}}+\frac{\partial^{2} \psi}{\partial y^{2}}=e^{\psi}
$$

the two-dimensional Liouville equation, which also possesses an infinitedimensional group which is easily derived from (3.6). Writing

$$
Z=\ln W, \quad W=u(x, y)+i v(x, y)
$$

(3.10) is equivalent to

$$
e^{\psi}=\frac{8\left\{\left(\frac{\partial u}{\partial x}\right)^{2}+\left(\frac{\partial u}{\partial y}\right)^{2}\right\}}{\left(u^{2}+v^{2}-1\right)^{2}},
$$

which is a standard form for a general solution to (3.11) (see, for example, Stuart [17] equation (5.6)). In the limiting case $f_{0} \rightarrow 0$, the solution (3.9) becomes $c=2 t /\left(x+x_{0}\right)^{2}$ which maps to

$$
c=2\left\{\left(\frac{\partial X}{\partial x}\right)^{2}+\left(\frac{\partial X}{\partial y}\right)^{2}\right\} t / X^{2} .
$$

In Dorodnitsyn et al [5], a very special case of the separable form $c=\operatorname{tg}(x, y)$ was considered. By using the global form of the group we are here immediately able to obtain the general case (3.10), together with the singular case (3.14). As a simple example, again choosing $Z=z^{1+\beta}$, reads

$$
c=\frac{2(1+\beta)^{2} t}{r^{2} \cos ^{2}(1+\beta) \theta} .
$$

We note that separable solutions of the form $c=\left(t_{0}-t\right) g(x, y)$ (where $t_{0}$ is a constant) can also be of interest. The solution in one dimension then reads

$$
c=\left(t_{0}-t\right) f_{0} / \cosh ^{2}\left\{\left(\frac{f_{0}}{2}\right)^{\frac{1}{2}}\left(x+x_{0}\right)\right\}
$$

and yields the two-dimensional solution

$$
c=\left\{\left(\frac{\partial X}{\partial x}\right)^{2}+\left(\frac{\partial X}{\partial y}\right)^{2}\right\}\left(t_{0}-t\right) / \cosh ^{2} X .
$$

Equivalently, writing $c=\left(t_{0}-t\right) e^{\psi(x, y)}$ now gives the Liouville equation

$$
\frac{\partial^{2} \psi}{d x^{2}}+\frac{\partial^{2} \psi}{\partial y^{2}}=-e^{\psi}
$$


and, using (3.13), (3.15) becomes

$$
e^{\psi}=8\left\{\left(\frac{\partial u}{\partial x}\right)^{2}+\left(\frac{\partial u}{\partial y}\right)^{2}\right\} /\left(u^{2}+v^{2}+1\right)^{2},
$$

which is equivalent to equation (5.4) of Stuart [17].

Several other exact solutions to (3.7) are also known, and these may be used in a similar fashion to derive new two-dimensional solutions.

$3.3 c_{t}=\left(c^{-\frac{4}{5}} c_{x}\right)_{x}+\left(c^{-\frac{4}{5}} c_{y}\right)_{y}+\left(c^{-\frac{4}{5}} c_{z}\right)_{z}$

We now turn to the three-dimensional equation (3.3). The results of Branson and Steeb [2], Dorodnitsyn et al [5] and Kersten and Gragert [11] show that there are three extra parameters in the invariance group of (3.3) compared to that of (3.1) in three dimensions with $n \neq-\frac{4}{5}$. We consider transformations (parametrised by $\varepsilon$ ) of the form

$$
\begin{gathered}
X=X(x, y, z ; \varepsilon), \quad Y=Y(x, y, z ; \varepsilon), \quad Z=Z(x, y, z ; \varepsilon), \\
T=t, \quad C=C(x, y, z, c ; \varepsilon),
\end{gathered}
$$

with corresponding infinitesimals $x^{*}, y^{*}, z^{*}, c^{*}$, where

$$
\begin{array}{rlrl}
X & \sim x+\varepsilon x^{*}(x, y, z), & & Y \sim y+\varepsilon y^{*}(x, y, z), \\
Z & \sim z+\varepsilon z^{*}(x, y, z), & C \sim c+\varepsilon c^{*}(x, y, z, c),
\end{array}
$$

as $\varepsilon \rightarrow 0$. The extra infinitesimals for (3.3) are given by
(a) $x^{*}=x^{2}-y^{2}-z^{2}, y^{*}=2 x y, z^{*}=2 x z, c^{*}=-5 x c$,
(b) $x^{*}=2 x y, y^{*}=y^{2}-x^{2}-z^{2}, z^{*}=2 y z, c^{*}=-5 y c$,
(c) $x^{*}=2 x z, y^{*}=2 y z, z^{*}=z^{2}-x^{2}-y^{2}, c^{*}=-5 z c$.

The global form of the group is given by the solution to

$$
\left.\begin{array}{rlrl}
\frac{d X}{d \varepsilon} & =x^{*}(X, Y, Z), \quad \frac{d Y}{d \varepsilon} & =y^{*}(X, Y, Z), \\
\frac{d Z}{d \varepsilon} & =z^{*}(X, Y, Z), \quad \frac{d C}{d \varepsilon}=c^{*}(X, Y, Z, C), \\
\text { ith } X & =x, Y=y, Z=z, C=c, \text { at } \varepsilon=0 ;
\end{array}\right\}
$$

see, for example, Bluman and Cole [3]. Considering (a), and defining

$$
r=\left(x^{2}+y^{2}+z^{2}\right)^{\frac{1}{2}}, \quad R=\left(X^{2}+Y^{2}+Z^{2}\right)^{\frac{1}{2}},
$$

(3.16) leads to

$$
\begin{array}{ll}
\frac{d Y}{d \varepsilon}=2 X Y, & \frac{d Z}{d \varepsilon}=2 X Z, \\
\frac{d R^{2}}{d \varepsilon}=2 X R^{2}, & \frac{d C}{d \varepsilon}=-5 X C,
\end{array}
$$


so that

$$
y Z=z Y, \quad y R^{2}=r^{2} Y, \quad y^{-\frac{5}{2}} C=c Y^{-\frac{5}{2}},
$$

and (3.16) also implies

$$
\frac{d}{d \varepsilon}\left(\frac{X}{Y}\right)=-\frac{R^{2}}{Y}
$$

so that

$$
y X=\left(x-\varepsilon r^{2}\right) Y .
$$

From (3.17) and (3.18) we may deduce, writing $\varepsilon=-\alpha$,

$$
\left.\begin{array}{ll}
X=\frac{x+\alpha r^{2}}{1+2 \alpha x+\alpha^{2} r^{2}}, & Y=\frac{y}{1+2 \alpha x+\alpha^{2} r^{2}}, \\
Z=\frac{z}{1+2 \alpha x+\alpha^{2} r^{2}}, & C=\left(1+2 \alpha x+\alpha^{2} r^{2}\right)^{\frac{5}{2}} c .
\end{array}\right\}
$$

The infinitesimals (b) and (c) lead to similar expressions and by combining all three groups we end up with

$$
\left.\begin{array}{l}
X=\frac{x+\alpha r^{2}}{1+2(\alpha x+\beta y+\gamma z)+\left(\alpha^{2}+\beta^{2}+\gamma^{2}\right) r^{2}}, \\
Y=\frac{y+\beta r^{2}}{1+2(\alpha x+\beta y+\gamma z)+\left(\alpha^{2}+\beta^{2}+\gamma^{2}\right) r^{2}}, \\
Z=\frac{z+\gamma r^{2}}{1+2(\alpha x+\beta y+\gamma z)+\left(\alpha^{2}+\beta^{2}+\gamma^{2}\right) r^{2}}, \\
C=\left(1+2(\alpha x+\beta y+\gamma z)+\left(\alpha^{2}+\beta^{2}+\gamma^{2}\right) r^{2}\right)^{\frac{5}{2}} c,
\end{array}\right\}
$$

where $\alpha, \beta$ and $\gamma$ are arbitrary constants. The relation

$$
R^{2}=\frac{r^{2}}{1+2(\alpha x+\beta y+\gamma z)+\left(\alpha^{2}+\beta^{2}+\gamma^{2}\right) r^{2}}
$$

also holds. We note that (3.20) corresponds to (3.19) combined with rotations.

If in (3.19) we replace $x$ by $-(1 / \alpha)-x$ and $X$ by $X+(1 / \alpha)$ (we note that we are exploiting the invariance of (3.3) under reflections of $x$ as well as translations) and set $\alpha=1$ we obtain the discrete transformation

$$
X=x / r^{2}, \quad Y=y / r^{2}, \quad Z=z / r^{2}, \quad C=r^{5} c
$$

which gives $R=1 / r$ and generalises (2.46) with $N=3$. It should be emphasised that (3.22) is not a special case of (3.20). It follows from (3.20) that if $c=g(x, t)$ is a solution to the one-dimensional problem

$$
\frac{\partial c}{\partial t}=\frac{\partial}{\partial x}\left(c^{-\frac{4}{3}} \frac{\partial c}{\partial x}\right)
$$


then

$$
\begin{aligned}
c= & \left(1+2(\alpha x+\beta y+\gamma z)+\left(\alpha^{2}+\beta^{2}+\gamma^{2}\right) r^{2}\right)^{-\frac{3}{2}} \\
& \times g\left(\frac{x+\alpha r^{2}}{1+2(\alpha x+\beta y+\gamma z)+\left(\alpha^{2}+\beta^{2}+\gamma^{2}\right) r^{2}}, t\right)
\end{aligned}
$$

is a solution to (3.3).

To give a simple example, we note that

$$
c=\left(\frac{4}{5} q(x-q t)\right)^{-\frac{5}{4}}
$$

where $q$ is an arbitrary constant, gives a travelling wave solution to (3.23). Setting $\beta=\gamma=0$, this maps to

$$
c=\left(1+2 \alpha x+\alpha^{2} r^{2}\right)^{-\frac{5}{2}}\left(\frac{4}{5} q\left(\frac{x+\alpha r}{1+2 \alpha x+\alpha^{2} r^{2}}-q t\right)\right)^{-\frac{5}{4}} .
$$

Applying the transformation (3.22) instead yields

$$
c=\left(\frac{4}{5} q r^{2}\left(x-q t r^{2}\right)\right)^{-\frac{5}{4}}
$$

which is equivalent to solution (11) of Yang et al [18] (with misprints corrected) and gives a more straightforward derivation of it.

Other well-known exact solutions to (3.23) can similarly be used to give three-dimensional solutions, as can (for example) solutions to (3.3) which depend only on $\left(x^{2}+y^{2}\right)^{\frac{1}{2}}$ and $t$.

\section{Discussion}

In this paper we have employed a technique of directly determining transformations which is more general than a Lie group approach in the sense that it identifies discrete as well as continuous transformations which map a given equation into itself as well as giving transformations to other equations of interest. An analogy may be made with the direct method of determining similarity solutions used by Clarkson and Kruskal [4], which finds nonclassical and well as classical self-similar solutions. We have clearly not considered the most general local transformations possible, but the ones we have considered seem the most general forms likely to give nontrivial results for the equations studied. The form of the transformations considered can easily be generalised, however, and this is necessary for linear diffusion $(n=m=0)$ 
where (2.6) and (2.7) become

$$
\begin{aligned}
x^{M} \frac{\partial u}{\partial t}=\frac{\partial^{2} u}{\partial x^{2}}, & N \neq 2, \\
e^{2 x} \frac{\partial u}{\partial t}=\frac{\partial^{2} u}{\partial x^{2}}, & N=2 .
\end{aligned}
$$

Making the more general transformation

$$
X=g(x, t), \quad T=h(t), \quad U=u / f(x, t),
$$

(4.1) yields

$$
\begin{aligned}
x^{M} f \frac{d h}{d t} \frac{\partial U}{\partial T} & =f\left(\frac{d g}{d x}\right)^{2} \frac{\partial^{2} U}{\partial X^{2}} \\
& +\left(2 \frac{\partial f}{\partial x} \frac{\partial g}{\partial x}+f \frac{\partial^{2} g}{\partial x^{2}}-x^{M} f \frac{\partial g}{\partial t}\right) \frac{\partial U}{\partial X}+\left(\frac{\partial^{2} f}{\partial x^{2}}-x^{M} \frac{\partial f}{\partial t}\right) U,
\end{aligned}
$$

so that we must impose

$$
x^{M} \frac{\partial f}{\partial t}=\frac{\partial^{2} f}{\partial x^{2}}
$$

and

$$
x^{M} f \frac{\partial g}{\partial t}=f \frac{\partial^{2} g}{\partial x^{2}}+2 \frac{\partial f}{\partial x} \frac{\partial g}{\partial x} .
$$

Writing $g=G / f$ and using (4.3), (4.4) becomes

$$
x^{M} \frac{\partial G}{\partial t}=\frac{\partial^{2} G}{\partial x^{2}} .
$$

We also require that

$$
x^{M} \frac{d h}{d t} /\left(\frac{\partial g}{\partial x}\right)^{2}
$$

be independent of $T$, and this requires that

$$
g=\omega\left(x^{\frac{\mu+2}{2}}\left(\frac{d h}{d t}\right)^{\frac{1}{2}}+g_{0}(t)\right),
$$

for some functions $\omega$ and $g_{0} . g$ must also satisfy (4.4), and this restricts the possible forms of $\omega, g_{0}$ and $h$. Similar results hold for (4.2) but we will not complete the analysis here.

The results of this paper show that the cases $n=-4 /(N+2)$ and $n=-2(N-3) /(N-4)$ are special and it is noteworthy that the two cases in which they coincide are $n=-4 / 3, N=1$, and $n=-1, N=2$.

In Section 3 we used the global forms of the transformations to derive new solutions which add significantly to the class of known genuinely multidimensional exact solutions to nonlinear diffusion equations. It is hoped 
that these solutions will provide useful test cases for codes for the numerical solution of such equations.

Our results can be extended in a number of ways. For example, a similar analysis has been performed on more general inhomogeneous nonlinear diffusion equations of the form

$$
F(x) G(u) \frac{\partial u}{\partial t}=\frac{\partial^{2} u}{\partial x^{2}}
$$

also giving new results.

Direct extensions of our solutions to other equations can be obtained using nonlocal transformations such as those given by King [13]. In particular, solutions to

$$
\frac{\partial c}{\partial t}=\frac{\partial}{\partial x}\left(D(c) \frac{\partial c}{\partial x}\right)
$$

may also be used to generate solutions to

$$
\frac{\partial v}{\partial t}=D\left(\frac{\partial v}{\partial x}\right) \frac{\partial^{2} v}{\partial x^{2}} \quad \text { and } \quad \frac{\partial w}{\partial t}=K\left(\frac{\partial^{2} w}{\partial x^{2}}\right)
$$

via the relations

$$
c=\frac{\partial v}{\partial x}, \quad v=\frac{\partial w}{\partial x}, \quad K(c)=\int^{c} D(\hat{c}) d \hat{c},
$$

and also solutions to

$$
\begin{aligned}
& \frac{\partial w^{*}}{\partial t^{*}}=K^{*}\left(\frac{\partial^{2} w^{*}}{\partial x^{* 2}}\right), \\
& \frac{\partial v^{*}}{\partial t^{*}}=D^{*}\left(\frac{\partial v^{*}}{\partial x^{*}}\right) \frac{\partial^{2} v^{*}}{\partial x^{* 2}}, \\
& \frac{\partial c^{*}}{\partial t^{*}}=\frac{\partial}{\partial x^{*}}\left(D^{*}\left(c^{*}\right) \frac{\partial c^{*}}{\partial x^{*}}\right),
\end{aligned}
$$

where

$$
\begin{gathered}
D^{*}\left(c^{*}\right)=D\left(1 / c^{*}\right) / c^{* 2}, K^{*}\left(c^{*}\right)=-K\left(1 / c^{*}\right), \text { and } \\
c^{*}=\frac{\partial v^{*}}{\partial x^{*}}=c^{-1}, \quad v^{*}=\frac{\partial w^{*}}{\partial x^{*}}=x, \quad x^{*}=v, \\
w+w^{*}=x x^{*}, \quad t^{*}=t .
\end{gathered}
$$

These transformations are described in more detail in King [13].

An analogous hierarchy of equations may be obtained in higher dimensions. Writing $c=\nabla \cdot \underset{\sim}{v}, \underset{\sim}{v}=\nabla w$ gives

$$
\frac{\partial \underset{\sim}{v}}{\partial t}=D(\nabla \cdot \underset{\sim}{v}) \nabla^{2} \underset{\sim}{v}, \quad \nabla \wedge \underset{\sim}{v}=0
$$


and

$$
\frac{\partial w}{\partial t}=K\left(\nabla^{2} w\right)
$$

The hodograph transformation

$$
{\underset{\sim}{v}}^{*}=\underset{\sim}{x}, \quad \underset{\sim}{x}=\underset{\sim}{v}, \quad t^{*}=t
$$

may be applied to (4.5) to obtain a problem for $v_{\sim}^{*}\left(\underset{\sim}{x^{*}}, t^{*}\right)$ and, equivalently, the Legendre transformation

$$
\nabla_{\sim}{ }_{\sim}^{*} w^{*}=\underset{\sim}{x}, \quad \underset{\sim}{x}=\nabla_{\tilde{x}} w, \quad w+w^{*}=\underset{\sim}{x} \cdot \underset{\sim}{x} x^{*}
$$

may be applied to (4.6), but the resulting equations seem unlikely to have physical application. We note in particular the case (3.2), when (4.6) reads

$$
\frac{\partial w}{\partial t}=\ln \left(\frac{\partial^{2} w}{\partial x^{2}}+\frac{\partial^{2} w}{\partial y^{2}}\right) \text {. }
$$

This has an infinite-dimensional group corresponding to that of (3.2); if $w(x, y, t)$ satisfies (4.7) then so does

$$
w(X, Y, t)+t \ln \left(\left(\frac{\partial X}{\partial x}\right)^{2}+\left(\frac{\partial X}{\partial y}\right)^{2}\right)
$$

provided that $X(x, y)$ and $Y(x, y)$ are harmonic conjugates. An equivalent result holds for the corresponding system of the form (4.5).

\section{Appendix: Conditions for the reduction of the dimensionality of initial-value problems for (3.2)}

We consider the Cauchy problem for (3.2), imposing the initial condition

$$
\text { at } t=0, \quad c=c_{0}(x, y) \text { for }(x, y) \in \mathbf{R}^{2},
$$

together with suitable conditions at infinity. We shall derive conditions which $c_{0}(x, y)$ must satisfy in order for (3.2) subject to (A.1) to have a solution which may be determined by solving the one-dimensional equation (3.7).

It is clear from (3.8) that if

$$
c_{0}(x, y)=\left\{\left(\frac{\partial X}{\partial x}\right)^{2}+\left(\frac{\partial X}{\partial y}\right)^{2}\right\} g_{0}(X)
$$

for some function $g_{0}$ and some harmonic $X(x, y)$, then (3.2) has a solution which satisfies (A.1) and takes the form (3.8) for all time, with $g(X, t)$ being determined from a solution $c=g(x, t)$ of (3.7) which satisfies the condition 
at $t=0, c=g_{0}(x)$. We note in passing that the form of solution (3.8) gives a similarity reduction for (3.2) resulting from the infinite-dimensional group.

We must therefore derive conditions on $c_{0}$ such that (A.2) is satisfied for some $g_{0}$ and some harmonic $X$. The conditions derived enable $g_{0}$ and $X$ to then be constructed. We first note that if (A.2) holds then, defining

$$
\omega(x, y) \equiv \frac{1}{c_{0}}\left(\frac{\partial^{2}}{\partial x^{2}}\left(\ln c_{0}\right)+\frac{\partial^{2}}{\partial y^{2}}\left(\ln c_{0}\right)\right),
$$

it follows that

$$
\omega(x, y)=\frac{1}{g_{0}} \frac{d^{2}}{d X^{2}}\left(\ln g_{0}\right)
$$

so that $\omega$ must be a function of $X$ only. Then if $\omega \not \equiv$ constant, we therefore write $X=X(\omega)$ and define

$$
\sigma(x, y) \equiv\left(\frac{\partial^{2} \omega}{\partial x^{2}}+\frac{\partial^{2} \omega}{\partial y^{2}}\right) /\left(\left(\frac{\partial \omega}{\partial x}\right)^{2}+\left(\frac{\partial \omega}{\partial y}\right)^{2}\right)
$$

It then follows that

$$
\sigma=-\frac{d^{2} X}{d \omega^{2}} / \frac{d X}{d \omega}
$$

For a given $c_{0}, \omega$ can be evaluated from (A.3) and then $\sigma$ from (A.5). Equation (A.6) requires that $\sigma$ depend on $x$ and $y$ only through $\omega$, and this provides the first condition that $c_{0}$ needs to satisfy in order that (A.2) hold. If this condition is met then (A.6) may be solved to give $X(\omega)$, and $X(x, y)$ is then known. We note that if $X_{1}(\omega)$ is one solution to (A.6) then the general solution is given by

$$
X(\omega)=k_{0}+k_{1} X_{1}(\omega)
$$

where $k_{0}$ and $k_{1}$ are arbitrary constants; $k_{0}$ represents a translation of $X$ and $k_{1}$ a rescaling so all solutions (A.7) are equivalent.

Once $X$ is determined the remaining condition to be satisfied is that

$$
\mu(x, y) \equiv c_{0}(x, y) /\left(\left(\frac{\partial X}{\partial x}\right)^{2}+\left(\frac{\partial X}{\partial y}\right)^{2}\right)
$$

depends on $x$ and $y$ only through $X$, and this gives $g_{0}(X)$. Hence the two conditions $c_{0}$ must satisfy are that:

(i) $\sigma$ depend only on $\omega$.

$\omega$ and $\sigma$ are calculated from $c_{0}$ by (A.3) and (A.5). Solving (A.6) with $\sigma(\omega)$ determined then gives $X(x, y)$.

(ii) $\mu$ depend only on $X$.

(A.8) defines $\mu$, and $g_{0}$ is then given by $g_{0}(X)=\mu$. 
The foregoing analysis breaks down if $\omega=\omega_{0}$, a constant, so that $\sigma$ is not defined. Then (A.3) reads

$$
\frac{\partial^{2}}{\partial x^{2}}\left(\ln c_{0}\right)+\frac{\partial^{2}}{\partial y^{2}}\left(\ln c_{0}\right)=\omega_{0} c_{0},
$$

and a solution is given by the separable form $c=\left(1+\omega_{0} t\right) c_{0}(x, y)$.

As a very simple illustration, we construct $X$ and $g_{0}$ in the radially symmetric case, which we have already shown may be reduced to a onedimensional problem. Writing $r^{2}=x^{2}+y^{2}, c_{0} \equiv c_{0}(r),(\mathrm{A} .3)$ reads

$$
\omega=\frac{1}{r c_{0}} \frac{d}{d r}\left(r \frac{d}{d r}\left(\ln c_{0}\right)\right)
$$

and $(\mathrm{A} .5)$ is

$$
\sigma=\frac{1}{r} \frac{d}{d r}\left(r \frac{d \omega}{d r}\right) /\left(\frac{d \omega}{d r}\right)^{2}
$$

Clearly $\omega$ and $\sigma$ are functions only of $r$, so that $\sigma$ depends on $x$ and $y$ only through $\omega$ and condition (i) is satisfied. (A.6) becomes

$$
\frac{d \omega}{d r} \frac{d^{2} X}{d \omega^{2}} / \frac{d X}{d \omega}=-\frac{1}{r}-\frac{d^{2} \omega}{d r^{2}} / \frac{d \omega}{d r}
$$

so

$$
\frac{d \omega}{d r} \frac{d X}{d \omega}=\frac{k_{1}}{r}
$$

and

$$
X=k_{0}+k_{1} \ln r .
$$

Setting $k_{0}=0, k_{1}=1$ gives $X=\ln r$ so that (A.8) reads $\mu=r^{2} c_{0}(r)$ which may be written as a function of $X$ only and condition (ii) is satisfied, giving $g_{0}(X)=e^{2 X} c_{0}\left(e^{X}\right)$. This gives the reduction mentioned in Section 1 .

\section{References}

[1] W. F. Ames, Nonlinear partial differential equations in engineering. Volume II. (Academic Press, New York, 1972).

[2] T. P. Branson and W-H. Steeb, "Symmetries of nonlinear diffusion equations", J. Phys. $A 16$ (1983) 469-472.

[3] G. W. Bluman and J. D. Cole, Similarity methods for differential equations. (SpringerVerlag, New York, 1974).

[4] P. A. Clarkson and M. D. Kruskal, "New similarity reductions of the Boussinesq equation", J. Math. Phys. 30 (1989), 2201-2213.

[5] V. A. Dorodnitsyn, I. V. Knyazeva and S. R. Svirshchevskii, "Group properties of the heat-conduction equation with a source in the two- and three-dimensional cases", Differential Equations 19 (1983) 901-908. 
[6] H. Fujita, "The exact pattern of concentration-dependent diffusion in a semi-infinite medium, part I", Text. Res. J. 22 (1952) 757-760.

[7] V. A. Galaktionov, V. A. Dorodnitsyn, G. G. Elenin, S. P. Kurdyumov and A. A. Samarskii, “A quasilinear heat equation with a source: peaking, localization, symmetry, exact solutions, asymptotics, structures", J. Sov. Math. 41 (1988) 1222-1292.

[8] D. L. Hill and J. M. Hill, "Similarity solutions for nonlinear diffusion-further exact solutions", J. Eng. Math. 24 (1990), 109-124.

[9] J. M. Hill, Solution of differential equations by means of one-parameter groups. (Pitman, Boston, 1982).

[10] J. M. Hill, "Similarity solutions for nonlinear diffusion-a new integration procedure", J. Eng. Math. 23 (1989) 141-155.

[11] P. H. M. Kersten and P. K. H. Gragert, "The Lie algebra of infinitesimal symmetries of nonlinear diffusion equations", J. Phys. $A 16$ (1983) L685-L688.

[12] J. R. King, "Exact similarity solutions to some nonlinear diffusion equations", J. Phys. A 23 (1990) 3681-3697.

[13] J. R. King, "Some non-local transformations between nonlinear diffusion equations", $J$. Phys. A 23 (1990) 5441-5464.

[14] A. A. Lacey, J. R. Ockendon and A. B. Tayler, "Waiting-time' solutions of a nonlinear diffusion equation”, SIAM J. Appl. Math. 42 (1982) 1252-1264.

[15] G. A. Nariboli, "Self-similar solutions of some nonlinear equations", Appl. Sci. Res. 22 (1970) 449-461.

[16] L. V. Ovsiannikov, "Group relations of the equation of nonlinear conductivity", Dokl. Akad. Nauk SSSR 125 (1959) 492-495.

[17] J. T. Stuart, "On finite amplitude oscillations in laminar mixing layers", J. Fluid Mech. 29 (1967) 417-440.

[18] Yang Qing-jian, Chen Xing-Zhen, Zheng Ke-jie and Pan Zhu-Liang, "Similarity solutions to three-dimensional nonlinear diffusion equations", J. Phys. A 23 (1990) 265-269. 